FERMILAB-CONF-97/418-T

hep-ph/9712427

December 1997

\title{
Flavor and CP Violations from Sleptons at the Muon Collider
}

\author{
Hsin-Chia Cheng \\ Fermi National Accelerator Laboratory \\ P.O. Box 500 \\ Batavia, IL 60510
}

\begin{abstract}
Supersymmetric theories generally have new flavor and CP violation sources in the squark and slepton mass matrices. They will contribute to the lepton flavor violation processes, such as $\mu \rightarrow e \gamma$, which can be probed far below the current bound with an intense muon source at the front end of the muon collider. In addition, if sleptons can be produced at the muon collider, the flavor violation can occur at their production and decay, allowing us to probe the flavor mixing structure directly. Asymmetry between numbers of $\mu^{+} e^{-}$ and $e^{+} \mu^{-}$events will be a sign for $\mathrm{CP}$ violation in supersymmetric flavor mixing.
\end{abstract}

Talk presented at the Workshop on Physics at the First Muon Collider and at the Front End of a Muon Collider November 6-9, 1997, Fermilab, Batavia, Illinois 
Weak scale supersymmetry (SUSY) is one of the most attractive candidates for physics beyond the standard model (SM). The discovery of superpartners of the SM particles is promising at the planned future colliders. If SUSY is discovered, measuring the masses and couplings of the superpartners will become the focus of study. Measurements of the superparticle couplings is essential for verifying supersymmetry [1], and the superpartner masses provide information of the origin of SUSY breaking and unification at high scales [2]. In most SUSY extension of the standard model, mass matrices of the fermions and their scalar superpartners are, however, not diagonal in the same basis. New flavor mixing matrices $W$, analogous to the CKM matrix, will appear at the gaugino-fermion-sfermion vertices. These new flavor mixing matrices may provide clues to the puzzle of the flavor structure, and therefore should also be important to study. At the muon collider and its front end, these new flavor mixing effects can be studied both indirectly through the rare flavor-changing process and directly by the slepton production if they are accessible. In this talk, we will discuss the power in probing the SUSY flavor mixings of the muon collider and compare the indirect and the direct probes. This work grew out from the studies done with N. Arkani-Hamed, J. L. Feng, and L. J. Hall [3, 四.

Lepton flavor, although conserved in the SM, is typically violated in most SUSY extension of the SM, since the scalar partners of the fermions must be given mass, and the scalar mass matrices are generally not diagonal in the same basis as the fermion masses. When we work in the mass eigenstates of both leptons and sleptons, the flavor mixing matrices $W$ will appear in gaugino/Higgsino vertices,

$$
\begin{aligned}
& \tilde{e}_{L i} W_{L i \alpha}^{*} \overline{e_{L \alpha}} \tilde{\chi}^{0}+\tilde{e}_{L i}^{*} W_{L i \alpha} \overline{\tilde{\chi}^{0}} e_{L \alpha} \\
& +\tilde{e}_{R i} W_{R i \alpha}^{*} \overline{e_{R \alpha}} \tilde{\chi}^{0}+\tilde{e}_{R i}^{*} W_{R i \alpha} \overline{\tilde{\chi}^{0}} e_{R \alpha},
\end{aligned}
$$

where the Latin and Greek subscripts are generational indices for scalars and fermions, respectively. Nontrivial $W$ matrices generate contributions to the rare flavor-changing processes, such as $\mu \rightarrow e \gamma$. The $\mu \rightarrow e \gamma$ rate is proportional to (simplifying to 2 generation mixing, $W_{11}=\cos \theta_{12}, W_{12}=$ 


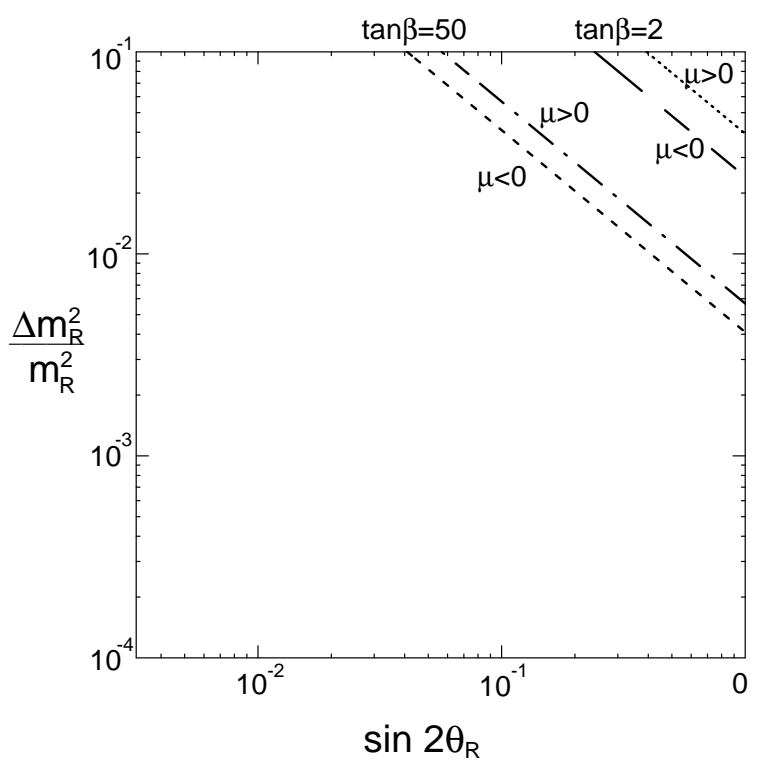

Figure 1: Constant contours of current bound on $B(\mu \rightarrow e \gamma)=4.9 \times 10^{-11}$ for the parameters given in the text and different $\tan \beta$ and signs of $\mu$.

$\left.\sin \theta_{12}\right)$

$$
\left(\frac{\Delta m_{12}^{2}}{\bar{m}_{12}^{2}} \sin 2 \theta_{12}\right)^{2},
$$

where $\bar{m}_{i j}^{2}=\left(m_{i}^{2}+m_{j}^{2}\right) / 2$ is the average squared slepton mass, and $\Delta m_{i j}^{2}=$ $\left(m_{i}^{2}-m_{j}^{2}\right) / 2 \approx 2 m \Delta m_{i j}$, with $\Delta m_{i j}=m_{i}-m_{j}$. It also depends on other SUSY parameters such as $m_{\tilde{\chi}_{0}}, \bar{m}_{L, R 12}, \mu, \tan \beta$, and so on. The current bound $B(\mu \rightarrow e \gamma)<4.9 \times 10^{-11}$ [5] puts strong constraints on $\frac{\Delta m_{12}^{2}}{\bar{m}_{12}^{2}}$ and $\sin 2 \theta_{12}$. The constraints on $\frac{\Delta m_{R 12}^{2}}{\bar{m}_{R 12}^{2}}$ and $\sin 2 \theta_{R 12}$ (assuming right mixing only) for $M_{1}=M_{2} / 2=130 \mathrm{GeV}, m_{\tilde{l}_{R}}=200 \mathrm{GeV}, m_{\tilde{l}_{L}}=350 \mathrm{GeV}$, and $|\mu|=$ $400 \mathrm{GeV}$ are shown in Fig. 11. Assuming no accidental cancellation among different diagrams, the $\mu \rightarrow e \gamma$ constraint requires $\frac{\Delta m_{12}^{2}}{\bar{m}_{12}^{2}} \sin 2 \theta_{12} \lesssim 10^{-2}$ for small $\tan \beta$, and about one order of magnitude stronger for large $\tan \beta(\sim 50)$. Therefore, if the mixing angle $\theta_{12}$ is not very small, $\tilde{e}$ and $\tilde{\mu}$ have to be quite degenerate in order to suppress the contribution to $\mu \rightarrow e \gamma$ by the superGIM mechanism.

Similarly, nonzero $W_{32}$ and $W_{31}$ can contribute to the rare decays $\tau \rightarrow \mu \gamma$ 
and $\tau \rightarrow e \gamma$. The current bounds $B(\tau \rightarrow \mu \gamma)<2.9 \times 10^{-6}, B(\tau \rightarrow e \gamma)<$ $2.7 \times 10^{-6}$ []], however, do not constrain the corresponding mixing angles and mass splittings for small $\tan \beta$, and constrain them only weakly for large $\tan \beta$. While $W_{32}$ and $W_{31}$ are not constrained individually, their product is constrained by $\mu \rightarrow e \gamma$ if large splitting between $\tilde{\tau}$ and $\tilde{\mu}$, $\tilde{e}$ masses exist, because $\mu \rightarrow e \gamma$ can occur through the $\tilde{\tau}$ loop.

At the muon collider, an extremely intense very low energy muon source will be available for greatly improving the search for rare muon decays. It is estimated that the bound on $B(\mu \rightarrow e \gamma)$ may be pushed down to $\sim$ $10^{-14}$ and even better for $\mu \rightarrow e$ conversion [6]. This will dramatically improve the probing range of $\frac{\Delta m_{12}^{2}}{\bar{m}_{12}^{2}}$ and $\sin 2 \theta_{12}$. The discovery of $\mu \rightarrow e \gamma$ will have important implications on the flavor structure and hence will be extremely exciting. However, the prediction of $B(\mu \rightarrow e \gamma)$ in SUSY theories still depends on many parameters and there are many diagrams which may add up or cancel each other. A single number $B(\mu \rightarrow e \gamma)$ is not enough for us to understand the whole flavor mixing matrices. We would like to get more handles on the $W$ matrices. At the muon collider, in addition to measuring $B(\mu \rightarrow e \gamma)$, if sleptons can be produced, we can probe these flavor mixing matrices directly from the flavor-changing slepton production and decay. They have simpler dependence on the SUSY parameters and there can be different modes to be measured. Therefore, they may provide more and clearer information for the flavor mixing matrices

We now consider the flavor-violating signals from on-shell slepton production at the muon collider. The signals we look for consist of a pair of unlike flavor leptons in the final state, $\mu^{+} \mu^{-} \rightarrow e_{\alpha}^{+} e_{\beta}^{-} \tilde{\chi}^{0} \tilde{\chi}^{0}$. For simplicity, let us consider the flavor-violating processes involving a single slepton as shown in Fig. 2. Summing over the amplitudes of different flavor sleptons, the cross section is proportional to

$$
\sum_{i j} W_{i \alpha} W_{i \beta}^{*} W_{j \alpha}^{*} W_{j \beta} \frac{1}{1+i x_{i j}},
$$

\footnotetext{
${ }^{1}$ The formalism for correlated slepton pair production is more complicated and is presented in [4]. However, the essential results remain unchanged.
} 


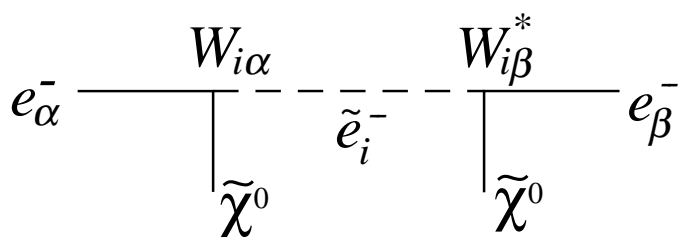

Figure 2: The flavor-violating process involving a single slepton production.

where $x_{i j} \equiv \Delta m_{i j} / \Gamma$, and $\Gamma$ is the slepton decay width. For two generation mixing, it reduces to (1-2 mixing only)

$$
\sin ^{2} 2 \theta_{12} \frac{x_{12}^{2}}{2\left(1+x_{12}^{2}\right)} \text {. }
$$

As with the low-energy signal, the flavor -violating collider signal vanishes in the limit of degenerate sleptons. However, the collider signal is suppressed only for $\Delta m<\Gamma$ where the quantum interference between different flavor sleptons becomes important, in constrast with the low energy signal which is suppressed by $\Delta m / \bar{m}$.

Having discussed the flavor-violating cross sections, we now consider the reach in the flavor mixing parameter space at the muon collider. We consider a case with the following SUSY parameters: $m_{\tilde{e}_{R}}, m_{\tilde{\mu}_{R}} \approx 200 \mathrm{GeV}, M_{1}=$ $M_{2} / 2=130 \mathrm{GeV}, \mu=-400 \mathrm{GeV}$, and $\tan \beta=2$. The LSP is almost pure bino and the cross section has little dependence on $\mu, M_{2}$, and $\tan \beta$ in this region. For center of mass energy $\sqrt{s}=500 \mathrm{GeV}$, we calculate the cross section of the flavor-violating signal $\mu^{ \pm} e^{\mp} \tilde{\chi}^{0} \tilde{\chi}^{0}$ as a function of $\sin 2 \theta_{12}$ and $\Delta m^{2}$, and the result is shown in Fig. 3. The major backgrounds come from $W W, W \nu \mu$, and $\tau \tau$ events. Assuming the similar cuts in the Next Linear Collider studies can be applied, the backgrounds can be reduced to $\sim 5$ $\mathrm{fb}$ while keeping about $30 \%$ of the signals. With the integrated luminosity $20 \mathrm{fb}^{-1}$, the $3 \sigma$ discovery limit is then $\sigma \sim 5 \mathrm{fb}$, which is shown by the thick contour in Fig. 3. We can see that $\sin \theta_{12}$ may be probed to $\sim 0.1$ for $\Delta m>\Gamma$. For comparison, we also superimpose the contours of the current $B(\mu \rightarrow e \gamma)$ constraint and the expected reach by the intense muon source at the muon collider front end. The collider probe reach far below the current 


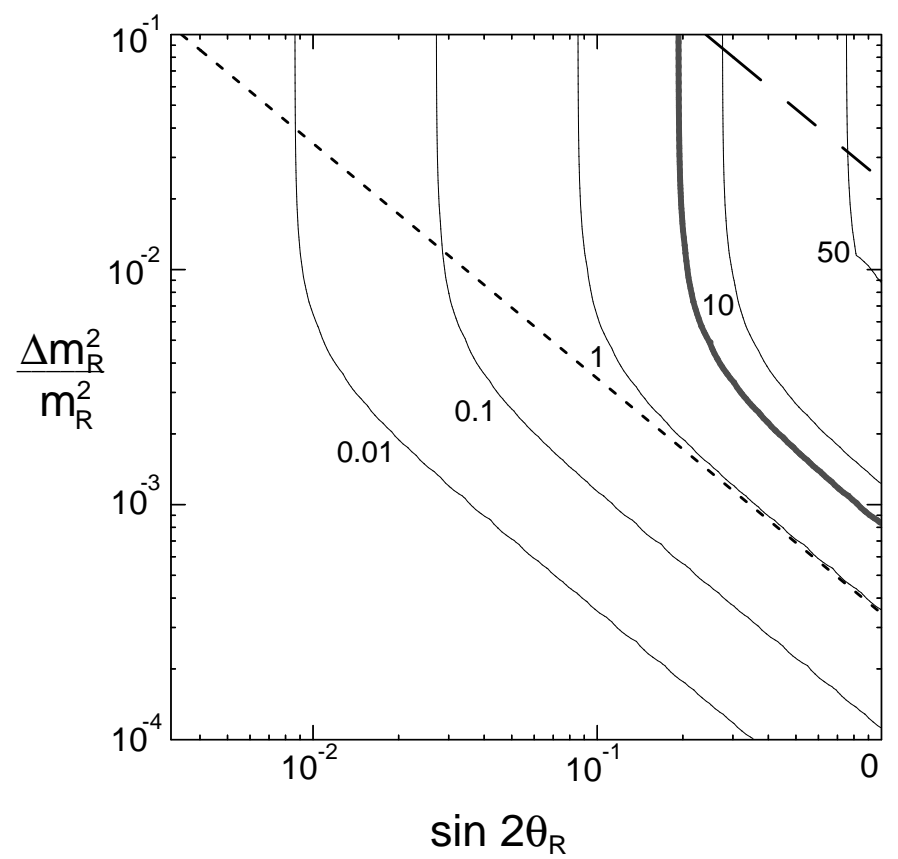

Figure 3: Contours of constant $\sigma\left(\mu^{+} \mu^{-} \rightarrow e^{ \pm} \mu^{\mp} \tilde{\chi}^{0} \tilde{\chi}^{0}\right)$ (solid) in fb for $\sqrt{s}=500 \mathrm{GeV}, m_{\tilde{e}_{R}}, m_{\tilde{\mu}_{R}} \approx 200 \mathrm{GeV}$, and $M_{1}=130 \mathrm{GeV}$. The thick contour represents the experimental reach with integrated luminosity $20 \mathrm{fb}^{-1}$. Constant contours of $B(\mu \rightarrow e \gamma)=4.9 \times 10^{-11}$ (dashed) and $10^{-14}$ (dotted) are also plotted for $m_{\tilde{l}_{L}} \approx 350 \mathrm{GeV}$. 
constraint for small $\Delta m$, but not as far as the expected $\mu \rightarrow e \gamma$ reach. However, $\mu \rightarrow e \gamma$ could receive contributions from many diagrams and has complicated dependence on many SUSY parameters. Therefore, it is more difficult to be disentangled to give precise information of flavor mixings than the collider signal.

It is also possible to probe 23 and 13 mixings by looking at final states of various flavor leptons. For the 23 mixing, the analysis is similar except that we look for final states with a $\tau$ instead of an $e$. Only hadronic decays of $\tau$ can be used as signals so the reach of the 23 mixing is a little worse than the 12 mixing. Nevertheless, it is still very interesting compared with no constraint from the current $\tau \rightarrow \mu \gamma$ bound. For the 13 mixing, because the dominant $t$-channel contribution to the slepton production always involves the initial state muons, we can only probed it (if possible) through the smaller $s$-channel contribution. Therefore, the probing power is not promising. In constrast, the electron collider can probe 13 mixing quite well, but not the 23 mixing.

The flavor mixing matrices may also contain $\mathrm{CP}$-violating phases. In the presence of $\mathrm{CP}$ violation, the cross sections $\sigma_{e_{\alpha}^{+} e_{\beta}^{-}}$and $\sigma_{e_{\beta}^{+} e_{\alpha}^{-}}$are no longer equal [4, 9], and the difference is proportional to the SUSY analogue to the Jarlskog invariant, $\tilde{J}$, which is defined by

$$
\operatorname{Im}\left[W_{i \alpha} W_{i \beta}^{*} W_{j \alpha}^{*} W_{j \beta}\right]=\widetilde{J} \sum_{k \gamma} \varepsilon_{i j k} \varepsilon_{\alpha \beta \gamma}
$$

Therefore, the asymmetry between the numbers of $\mu^{+} e^{-}$and $e^{+} \mu^{-}$events provides a signal for the $\mathrm{CP}$ violation in the slepton flavor mixing matrices. This CP-violating asymmetry can only be significant for large 3 generation mixings and the mass splittings among different generations comparable to $\Gamma$. Therefore, if it is discovered, it points toward a very specific flavor structure. Fig. 4 shows the $3 \sigma$ discovery limit of the CP asymmetry for the set of SUSY parameters: $m_{\tilde{l}_{R}}=150 \mathrm{GeV}, M_{1}=M_{2} / 2=100 \mathrm{GeV}, \mu=-400 \mathrm{GeV}$, $\tan \beta=2$, and assuming $\Delta m_{12}=\Delta m_{23} \equiv \Delta m, \theta_{12}=\theta_{23}=\theta_{13} \equiv \theta$, $\sin \delta=1$ in the standard parametrization [10].

In conclusion, if supersymmetry is discoverd, there will be a long and 


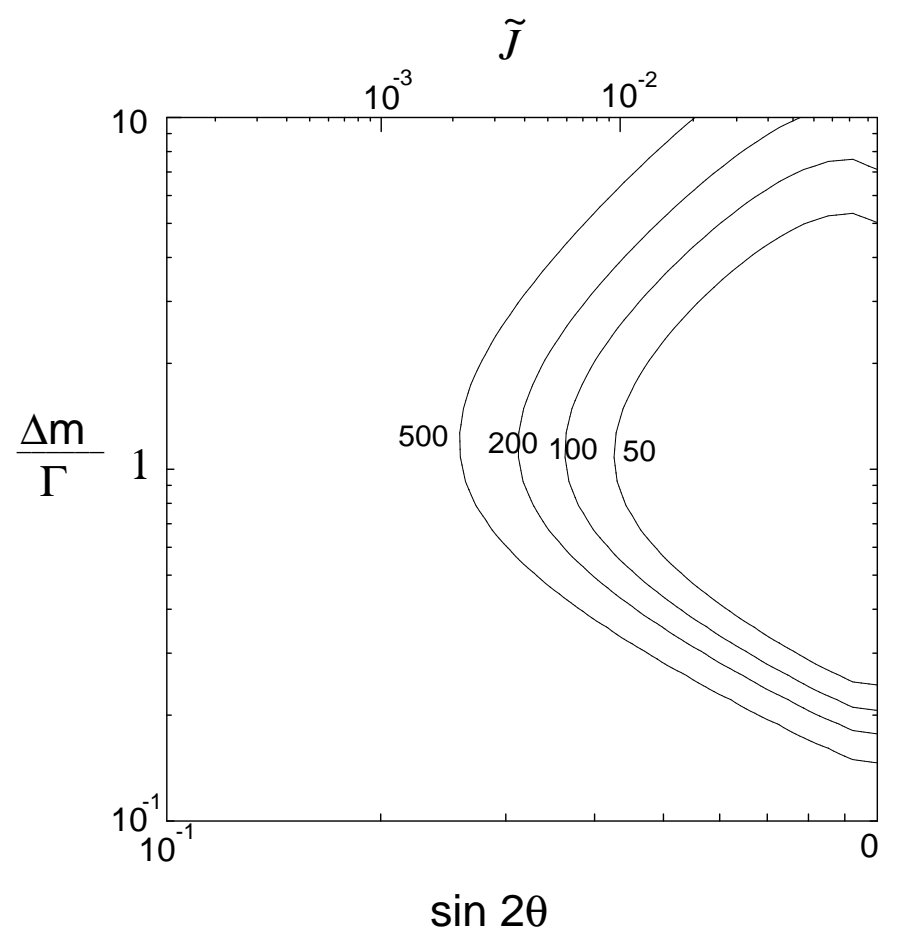

Figure 4: $3 \sigma$ slepton $\mathrm{CP}$ violation discovery contours for the integrated luminosity given $\left(\right.$ in $\mathrm{fb}^{-1}$ ). The $\mathrm{CP}$-violating phase is fixed to $\sin \delta=1$. The SUSY parameters are as given in the text. 
exciting road to measure the SUSY parameters in order to understand the underlain theory in nature. The SUSY flavor mixing matrices may provide important clues to the flavor structure and hence should be an important subject to study. At the muon collider, the flavor violation can be probed both by the low energy processes at the front end and direct slepton production at the collider. They can provide complementary information on the flavor mixings of the underlain SUSY theory.

\section{References}

[1] J. L. Feng, H. Murayama, M. E. Peskin and X. Tata, Phys. Rev. D52, 1418 (1995); M. M. Nojiri, K. Fujii and T. Tsukamoto, Phys. Rev. D54, 6756 (1996); H.-C. Cheng, J. L. Feng and N. Polonsky, Phys. Rev. D56, 6875 (1997), and Fermilab-PUB-97/205-T, hep-ph/9706476, to be published in Phys. Rev. D.

[2] S. Martin and P. Ramond, Phys. Rev. D48, 5365 (1993); Y. Kawamura, H. Murayama and M. Yamaguchi, Phys. Lett. B324, 52 (1994); H.-C. Cheng and L. J. Hall, Phys. Rev. D51, 5289 (1995); S. Dimopoulos, S. Thomas and J. D. Wells, Nucl. Phys. B488, 39 (1997).

[3] N. Arkani-Hamed, H.-C. Cheng, J.L. Feng and L.J. Hall, Phys. Rev. Lett. 77, 1937 (1996).

[4] N. Arkani-Hamed, H.-C. Cheng, J.L. Feng and L.J. Hall, Nucl. Phys. B505, 3 (1997).

[5] R.D. Bolton, et al., Phys. Rev. D38, 2077 (1988).

[6] W. Marciano, in these proceedings.

[7] CLEO Collaboration, K. W. Edwards et al., Phys. Rev. D55, 3919 (1997). 
[8] R. Becker and C. Vander Velde, in Proceedings of the European Meeting of the Working Groups on Physics and Experiments at Linear $e^{+} e^{-}$ Colliders, ed. P.M. Zerwas, Report No. DESY-93-123C, p. 457.

[9] D. Bowser-Chao and W.-Y. Keung, Phys. Rev. D56, 3924 (1997).

[10] L.-L. Chau and W.-Y. Keung, Phys. Rev. Lett. 53, 1802 (1984). 